\title{
EDITORIAL Contralateral transfalcine approach for peritrigonal lesions
}

\author{
Roberto C. Heros, MD \\ Department of Neurosurgery, University of Miami, Florida
}

$\mathrm{T}$ HIS issue of the Journal of Neurosurgery contains an excellent and beautifully illustrated article by Dr. Bohnstedt and colleagues describing their experience with the contralateral approach to peritrigonal lesions in 14 patients. ${ }^{1}$ I will begin by congratulating the senior author, Dr. Cohen-Gadol, for his outstanding surgical results in these patients, several of whom harbored very challenging lesions. Although there was the expected number of significant complications from the excision of the lesion, it is notable that only one complication, a transient leg weakness, could be directly attributable to the use of the contralateral approach. This obviously is attributable to the skill of the surgeon and perhaps a fair amount of good luck in terms of the number, location, and significance of the parasagittal draining veins in the region of the approach in their particular patients. I personally have found considerable difficulty finding an appropriate "corridor" between draining veins in this important postcentral region, and I frequently have had to work through an excessively narrow corridor to avoid sacrificing one of these important veins; in one instance I had to abandon the attempt and use a different surgical approach.

The authors do an excellent job of describing in a very understandable manner the advantages of this approach. By entering the region of the trigone medially, one avoids interruption of the optic radiations that course along the lateral wall of the trigone. The contralateral approach avoids retraction of the affected hemisphere, which may be edematous depending on the nature of the pathology. Clearly, the contralateral approach offers a more perpendicular view towards the lesion, which allows easier access to the superior and lateral aspect of the lesion as compared with the ipsilateral interhemispheric route that requires a tangential approach. It is also true that with arteriovenous malformations (AVMs) the contralateral approach facili- tates access to feeders from the choroidal arteries, which would require a more tangential approach with the ipsilateral interhemispheric route. However, the contralateral approach would require a considerably larger falcine window to afford the good early control of posterior cerebral artery feeders offered by the ipsilateral interhemispheric approach. Another important advantage of the contralateral approach is that, because the involved hemisphere is not manipulated, there is less likely to be "brain shift" on that hemisphere and therefore neuronavigation should be more accurate, which is important because as the authors emphasize, neuronavigation is very useful in the contralateral approach.

The potential pitfalls of the contralateral approach are also discussed by the authors, although perhaps not as emphatically as they discuss the advantages. The basic problem, of course, is the potential for bihemispheric damage, injuring the involved hemisphere during the resection of the lesion and the uninvolved hemisphere by the approach. As alluded to before, the greatest threat to the uninvolved hemisphere is offered by the parasagittal draining veins in the postcentral region. The more anterosuperior the interhemispheric approach, the shorter the route to the lesion and the better the exposure of the anterior aspect of the lesion. On the other hand, as the surgeon moves his approach anterosuperiorly, he will encounter more numerous and more significant draining veins that can limit the approach and make it more hazardous. The more posterior (occipital) approach generally avoids the problem with the parasagittal draining veins that are scarce and sometimes absent in this region; however, from a more posterior approach, the distance to the lesion is greater, the line of vision more tangential, and the risk of retraction injury to the occipital visual cortex greater. One of the most serious potential complications of this contralateral approach 
is a bilateral visual field defect. The optic radiations on the side of the lesion can be damaged during the resection, particularly with larger lesions that reach the lateral wall of the trigone. The ipsilateral visual cortex can be injured from retraction or from a venous infarction that could result from a damaged or sacrificed draining vein. The other obvious potential limitation of the contralateral approach is the need to work through a limited window in the falx, which may impair the surgeon's ability to deal with complications such as an unexpected intracerebral hemorrhage during resection of the lesion. I have covered in more detail the limitations of this approach in a recent editorial. ${ }^{3}$

The authors give us very useful "surgical pearls" to keep in mind when using this approach and I would like to emphasize some of these. I agree with the use of the semi-prone position, with the patient either on the lateral or park-bench position, and importantly, with the site of the approach down to allow gravity to help with the exposure. In addition to this, as the authors also point out, the use of CSF drainage by a lumbar drain or an intraoperative temporary ventriculostomy is, in my opinion, essential in these cases because there is no early access to CSF and relaxation is extremely important to avoid retraction damage. The authors discuss the importance of not injuring the straight sinus during the opening of the falx. They imply that at times it may not be perfectly straightforward to be certain of the location of the straight sinus by telling us that in 2 patients they used fluorescein angiography to confirm its location. I will emphasize the fact that it is easy to be wrong or uncertain about the location of the straight sinus. I have encountered this problem in a few occasions using the paraoccipital transtentorial approach to the pineal region. Even though the textbooks appear to show a clear right angle between the falx and the tentorium, this is frequently not the case and that angle is more curved and less obvious, which makes the determination of the location of the straight sinus sometimes difficult. Every precaution, including the use of neuronavigation and fluorescein angiography if necessary, should be used to avoid extending the incision of the falx into the straight sinus. Another potential problem that apparently the authors did not encounter is the presence of important venous channels along the falx between the sagittal and the straight sinuses. This can particularly be a serious problem in cases of AVMs in which draining veins may drain into the falx. Catastrophic hemorrhage, which may be very difficult to control, could be encountered during the opening of the falx if appropriate care is not taken. The authors also allude to the problem of injuring the optic radiations along the lateral wall of the trigone during resection of the lesion, and to this effect I agree with the recommendation of not using this approach for larger lesions that reach the lateral wall of the trigone. In terms of the craniotomy, my advice is to use a craniotomy with a broader base along the sagittal sinus than that illustrated by the authors. A broader base craniotomy allows the surgeon to choose the optimal corridor for the approach between draining veins. Maybe I am not good at interpreting MR images and even MR venograms, but I find that they are not entirely reliable in helping me predict the exact location of the veins.
My final piece of advice is to do a much "shallower" dural opening than that illustrated by the authors. A shallower opening allows the brain to fall away under the dura rather than falling against the dura, which could lead to cortical injury when the dural opening is too deep.

The authors discuss well the pros and cons of all the alternative approaches with the exception of the posterior parietal lobule approach, which, in fact, has been my preferred approach for AVMs involving this region and for large tumors such as meningiomas of the trigone. In my experience, this transcortical approach, which ideally spares the primary sensory radiations and the visual association fibers, can be used without either a resulting motor or sensory deficit or a visual field defect. ${ }^{2}$ Nowadays with neuronavigation this approach, for which I usually make a cortical incision located approximately $9 \mathrm{~cm}$ above the inion and about 3 to $4 \mathrm{~cm}$ lateral to the midline, can be used with considerable ease and safety and should always be kept in mind as a possible alternative to lesions in the trigone and paratrigonal region, particularly when they are located superiorly and medially. For lesions located inferior to the trigone, I have usually used an inferior temporal gyrus transcortical approach. These transcortical approaches do carry some risk of seizures but they avoid the risk of venous damage and minimize the need for brain retraction that is necessary, whether by fixed retractors or by "dynamic intermittent retraction," when using the interhemispheric approach.

I will finish by confessing that I have used this posterior contralateral approach only infrequently and then usually for cavernomas located medial and anterior to the trigone. However, I believe that this is a very reasonable and possibly preferred approach for small circumscribed tumors and perhaps even for small straightforward AVMs of this region. To approach more complex AVMs and larger tumors in this area with safety would require the experience and technical excellence for which Dr. CohenGadol is well known.

One of my colleagues in Miami, whom I consider a world-class master neurosurgeon, would forewarn our residents that when using this approach "the patient will be fine if the surgeon does everything right." Gradually through the years, I have learned that we do not always do everything right and have come to pay more attention to the teachings of my mentor, Bob Ojemann, who took all the precautions necessary in case anything that could go wrong would go wrong. This would include always using the safest possible surgical approach as opposed to the most elegant and challenging.

http://thejns.org/doi/abs/10.3171/2014.10.JNS142002

\section{References}

1. Bohnstedt BN, Kulwin CG, Shah MV, Cohen-Gadol AA: Posterior interhemispheric transfalcine transprecuneus approach for microsurgical resection of periatrial lesions: indications, technique, and outcomes. J Neurosurg [epub ahead of print May 1, 2015. DOI: 10.3171/2015.3.JNS14847]

2. Heros RC: Brain resection for exposure of deep extracerebral and paraventricular lesions. Surg Neurol 34:188-195, 1990

3. Heros RC: Editorial. Contralateral approach to the atrium of the lateral ventricle. J Neurosurg 113:947-948, 2010 


\section{Response}

\section{Aaron A. Cohen-Gadol, MD, MSc}

Goodman Campbell Brain and Spine, Indiana University Department of Neurological Surgery, and IU Simon Cancer Center, Indianapolis, Indiana

I sincerely appreciate the thoughtful comments of Dr. Heros. Only a few neurosurgeons are considered masters of complex cranial surgery, and Dr. Heros represents the best of them. His comments have elevated the value of our work and I am delighted and honored to read his editorial.

Dr. Heros has elegantly described the advantages and drawbacks of the posterior interhemispheric transfalcine transprecuneus approach (PITTA). It is important to emphasize the technical complexity of the PITTA due to its challenging, oblique, long working angles and depth, unfamiliar anatomy, and additional handling of the normal hemisphere. This alternative route should be used selectively and judiciously; otherwise, the risk of complications is likely to limit its adoption. Furthermore, the possibility of torrential bleeding through this corridor requires certain expertise in vascular neurosurgery.

One may question the rationale behind using more technically challenging operative routes in place of more traditional proven corridors. Would the patient be exposed to unnecessary risks? Would the technical complexity of the operation and associated risks justify the benefits of less brain transgression? There are many descriptions of new alternative approaches to difficult-to-reach tumors that claim superiority compared with well-described routes.
I myself struggle at times to find answers to these imperative questions, but I believe the justification depends on numerous factors, including the surgeon's comfort level with microsurgery and the special features of the pathology at hand. Ultimately, the best strategy for reaching a deep-seated lesion is determined by appropriate patient selection and reliable, unbiased, nondogmatic understanding of the risks and benefits of each operative approach.

One factor is worth further attention. "Neurosurgical dogma" should be carefully scrutinized. Surgical technique, craft, or finesse is not a static art, but defines the dynamic and progressive science of operative neurosurgery. The search for alternative operative routes to minimize brain transgression and retraction while maximizing lesional resection at the expense of operative technical complexity is the sine qua non of our innovative philosophy to advance the field of microsurgery. The operator's honesty will dictate if a relative increase in the level of technical complexity can lead to additional risk to the patient.

In summary, the PITTA is founded on the concept of using a contralateral operative trajectory to augment a more tangential working angle to the more difficult-toreach lateral target through a midline route. The PITTA emphasizes the importance of operative working angles (vs necessary operative space) through less disruptive exposures as a more viable parameter to achieve desirable results.

I want to again thank Dr. Heros for his magnificent and unparalleled contributions to the field of operative neurosurgery. 